\title{
Mobile Personalized Notes Using Memory Package
}

\author{
Cheng-Chieh Chiang \\ Department of Information Technology \\ Takming University of Science and Technology \\ No. 56, Sec. 1, Huan-Shan Rd., Taipei 114, Taiwan
}

\begin{abstract}
Smart phones and their mobile application have been deeply affected people life in the modern society. We can find many kinds of mobile applications, either in Google Play or Apple Store, designed according to novel motivations to assist us in improving people life. This paper aims to develop a personalized note tool on smart phones to integrate different types of multimedia information to help the users record activities. In this paper we propose a structure called memory package for integrating multimedia information on smart phones. Users can create a memory package in a time period to record their activities by using multimedia contents such as text, image, video, audio, and GPS records. Our system designs a unified interface for the memory package that can help users easily generate and review their packages on phones. Users can preserve clips of user activities of life anytime and anywhere using the proposed system of personalized notes as well as the memory package.
\end{abstract}

Keywords: memory package; personalized notes; smart phone; mobile application; multimedia information

\section{INTRODUCTION}

Smart phones have been more popular in the modern city. Many applications on smart phones, either in Android or iOS systems, are developed with different goals to deeply change our life. Let us take a simple case for illustration. When people take a smart phone, they can capture photos of interest in life, track the GPS trajectory of their family tour, or record an important meeting in work. In past, some applications have been designed to help the users efficiently utilize sensors of mobile devices, and hence kinds of multimedia information have been generated for different goals. How to well integrate mounted sensors as well as multimedia information is a critical issue to improve the utilities of mobile devices for users.

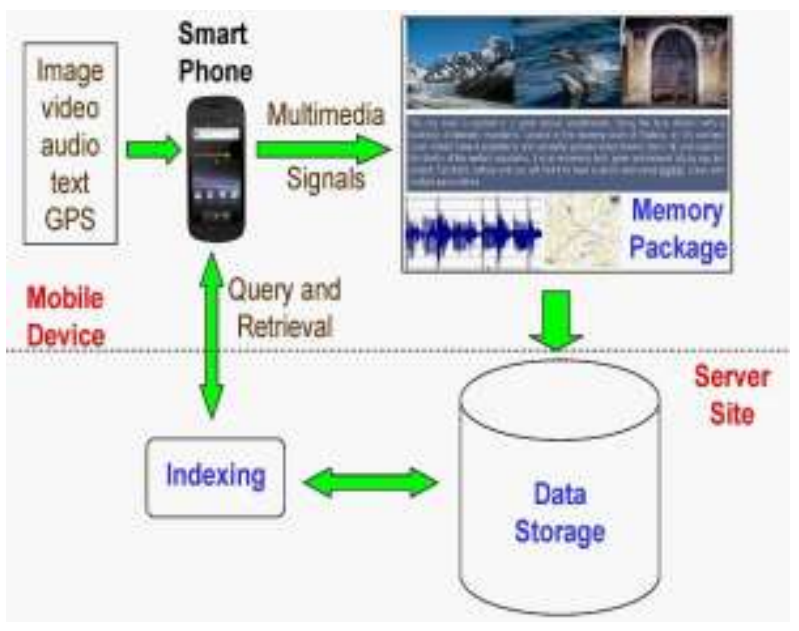

Figure. 1 The overall concept of our proposed ideas on smart phones.

In this paper we aim to design a mobile application that can serve people as a personalized secretary tool on their smart phones to integrate different types of multimedia information to help the users record activities in life. Figure 1 presents the overall concept of our proposed ideas how to integrate multimedia information to record human activities in smart phones. Here multimedia information including image, video, and audio can be captured by hand-held smart phones to assistant the users in keeping their activities anytime and anywhere. In our design, these multimedia information generated in our life are collected and called "memory package". Users can recall their memories in the package pool in this mobile application.

Let us take the following illustrations for our proposed application. Assume John attends a two-hour meeting of the supplier presentation. He launches our proposed application in smart phone and select "meeting" scene to start on a new memory package. First, John sets the sound recording on in order to record all discussions. While this meeting is running, John can have notes for his ideas and capture photos of products. This memory package involves two-hour contents of this meeting, including audios of the presentation and discussion, images of highlighting products, and textual notes of relate ideas for this meeting. In a happy weekend, John enjoys a vacation in beach with family. Our application starts again and set as "tour" scene to create another new memory package before leaving. The GPS trajectory during this tour can be preserved in this memory package. During this twoday vacation, John can record videos for his family, take pretty images for beautiful scenery, and write some notes for funny jokes. Then, the tour memory package stores his lovable memory in two days.

The design of the memory package can achieve several properties. First, one package can collect memory clips that are related to the same event, one to the meeting and the other to the family tour in our illustrations mentioned above. Moreover, these memory clips are constructed by different kinds of multimedia information and then integrated in the packages. Beside raw records such as recording audio and storing GPS sequences, the users can also highlight contents in the packages by writing notes and taking images according their preferences. Note that the selection of the scene mode provides a prior concept too. Then, the memory packages can be delivered to the server site and the advanced analysis can be performed based on the integration of multimedia memory. 
The rest of this paper is organized as the follows. Section 2 provides previous works related to this work. Section 3 presents our design of personalized memory package in this paper. Section 4 demonstrates the implementation details of the proposed system. Then finally, Section 5 makes our conclusions about this work and draws future works to improve this system.

\section{RELATED WORKS}

Personalized multimedia system has become more public in research and industrial areas in recent due to the rapid increasing of mobile devices. J. Martin and C. Trummer [7] proposed a personalized multimedia system for museums and exhibitions in a hand-held system. This system can adjust individual visitors' interests to refine the presentation of digital contents that are associated with knowledge based exhibitions and exhibition components. S. Potter et al. [8] designed a portable system called MediaPod that allows users maintaining the persistent and personalized multimedia environment on any available computer. W.-T. Chu et al. [2] proposed a tiling slideshow system to analyze video and audio information. The tiling slideshow system categorized video contents and then designed a playing scheme integrated with the audio tempo. K.-Y. Cheng et al. [1] proposed a video playing system called SmartPlayer to provide layers of events for users by analyzing motion and semantics in video. These two systems were designed for desktop or laptop, not for mobile system.

N. Sebe and Q. Tian [9] published a literature survey related to personalized multimedia retrieval in 2007. In their categorization, the possible future directions of personalized multimedia retrieval included the multimedia content description, user preferences and context, automatic content annotation, automated presentation authoring, and distributed retrieval and filtering of content descriptions and user preferences. J. Huber et al. [4] designed seven navigation approaches for video browsing on mobile device and deeply discussed the design space and the characteristics of interaction concepts for mobile video browsing. T. Karrer et al. [5] presented a mobile video system called PocketDRAGON that allows direct manipulation video navigation on mobile touch-screen devices.

In industry, many personalized image or video systems have been published in the past. For example, iPhoto [12] and iMovie [11] are two famous tools published by Apple. iPhoto can provide variant utilities for personal photos such as special-effect editing, sliding show, streaming, and event- or location-based photo management. iMovie focuses on video editing, including personal video generation, editing, template, special effect, etc. Users of Both of iPhoto and iMovie can publish their own photos and videos in Internet by use of Facebook and YouTube. Moreover, Apple also integrates these two systems with iCloud [10] such that all of images and videos can be handled over the clouding environment.

\section{MEMORY PACKAGE}

Smart phone is convenient to serve that people can record anything in anytime and anywhere. Multiple devices, such as camera and voice recording, are mounted in a smart phone to provide multimedia information for users. Thus, we always have much information stored in our smart phones such as images and videos etc., and then the information could be considered clips of our memory that can keep parts of life. Hence we design a unified structure called memory package to collect different types of multimedia information associated with our memory in life.

One of the main goals for memory package is to deal with the integration between different types of multimedia in a smart phone. Figure 2 presents the description layer of the memory package by providing extra metadata for multimedia contents. Metadata in the description layer includes the scene mode selected by the user, the temporal information, and the original metadata of multimedia. The temporal information contains the time order of multimedia, and the original metadata contains the corresponding information of image, video, and audio such as resolution and size etc. That is to say, the description layer aims to summarize multimedia contents included in the memory package. Moreover, our proposed system designs a unified interface to control multimedia devices (camera, voice recording, GPS) mounted in a smart phone to generate contents of the memory package. The users can employ our system to easily switch to these devices to record activities in life.

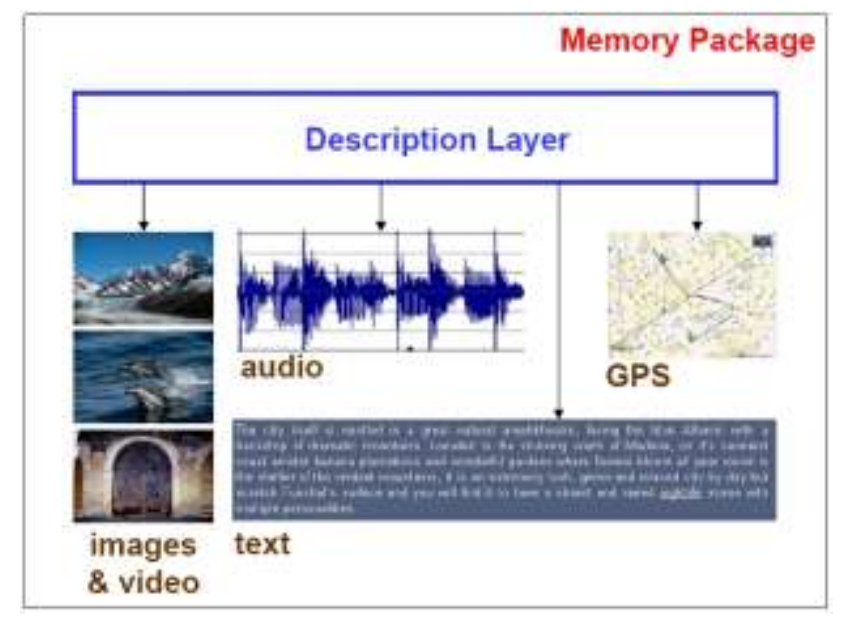

Figure. 2 The structure of the memory package. A description layer is appended to provide extra metadata of the corresponding multimedia contents.

When a new memory package is created, semantic relationships have been implicit in all multimedia contents. At least, all contents are associated with the same memory event. Indeed, there are also additional metadata in the description level that can help us construct semantic links among multimedia in the memory package. Furthermore, contentbased approach [3][6] can be applied to discover relationship of multimedia information across memory packages. Hence, our design of the memory package can provide a flexible structure that has potential to construct semantic connections both inter- and outer-packages.

\section{IMPLEMENTATION}

We have implemented a beginning version of the personalized application for memory package in smart phone. Our system was implemented in Android smart phones, and we hope to extend it to Apple iOS system. This section briefly introduces our implementation of the proposed personalized memory package in Android system. When the application is launched, the users have two choices in the home screen shown in 
Figure 3: new package and view package; the former to create a new memory package and the latter to browse and view existed packages.

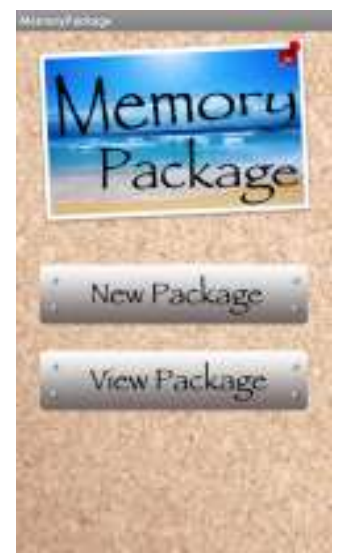

Figure. 3 Users can create and browse memory packages in the home page of our system.

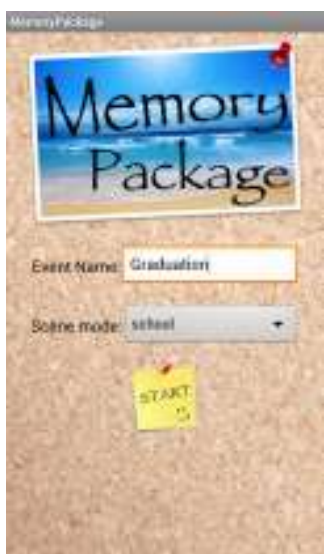

(a)

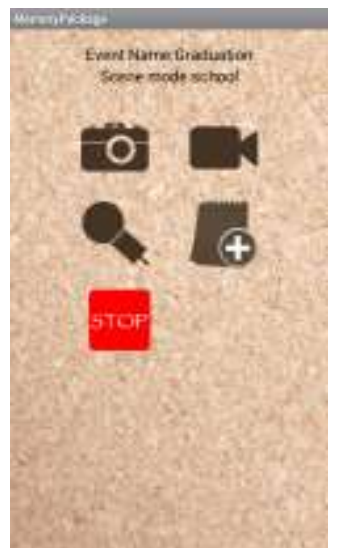

(b)

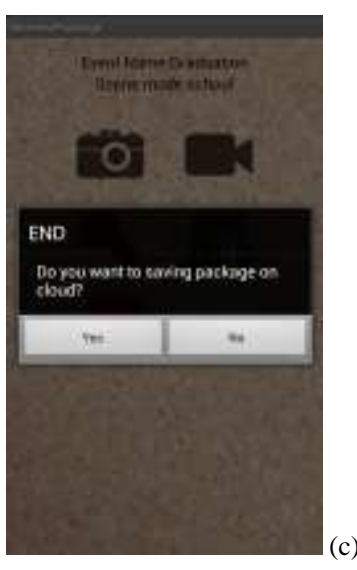

Figure. 4 The creation of a new memory package. (a) Set the package name and its corresponding scene mode. (b) Several multimedia devices can be used for recording human activities. (c) Make a copy to the storage on cloud when the new package has been created.

Figure 4 shows some snapshots of functions for creating new memory packages. When a new package is generated, a package name and its corresponding scene mode are first requested shown in Figure 4(a). Then, the users can select and switch multimedia devices to record anything during the package period, shown in Figure 4(b). Moreover, each of packages can be duplicated to make a copy to the storage on cloud shown in Figure 4(c) when this package creating is stopped.

The other main function, browsing packages shown in Figure 5 , aims to provide an easy way for the users to find packages of interest and then look at the detailed contents of multimedia. Figure 5(a) presents several browsing functions of memory packages, containing filtered by scene modes, sorted by name/time/scene, and displayed by icon/list. When the users select a specific package, its multimedia contents are listed shown as Figure 5(b). All of multimedia contents in a package are ordered by creation time, so the users can realize the temporal relationships among events of this memory package. The users can click each of elements in Figure 5(b) to view individual media files directly. Note that the first text file is the description of this package, and GPS coordinates can be appended into multimedia contents

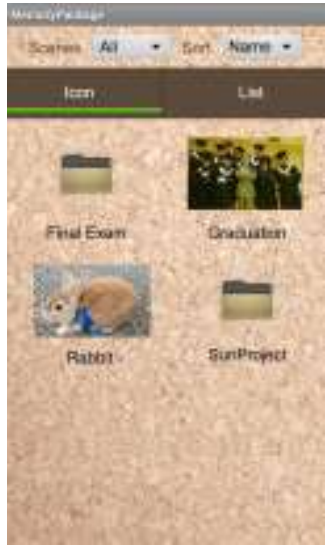

(a)

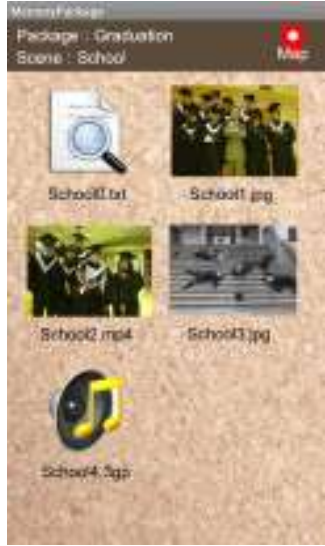

(b)
Figure. 5 The browsing of memory packages. (a) Several functions for browsing memory packages. (b) The multimedia contents of a memory package.

\section{CONCLUSION}

This paper presents our design of the personalized multimedia system to assist the users in recording life activities anytime and anywhere in smart phone. We propose an integrated structure called memory package to collect all of multimedia information captured by devices mounted in smart phone, such as image, video, audio, text, and GPS. This paper also describes the details of our proposed application that has been implemented in Android system. Future work mainly extends functions for our proposed system that only has the basic functions of our design at the current stage. Also, an advanced integration scheme should be considered to better visualize and represent multimedia information in memory packages.

\section{REFERENCES}

[1] K.-Y. Cheng, S.-J. Luo, B.-Y. Chen, and H.-H. Chu, "SmartPlayer: User-Centric Video Fast-Forwarding," in Proceedings of 27th ACM Conference on Human Factors in Computing Systems (CHI), 2009. 
[2] W.-T. Chu, J.-C. Chen, and J.-L. Wu, "Tiling Slideshow: An Audiovisual Presentation Method for Consumer Photos," IEEE Multimedia, 14(3), 2007.

[3] R. Datta, D. Joshi, J. Li, and J. Z. Wang, "Image Retrieval: Ideas, Influences, and Trends of the New Age," ACM Computing Surveys, Vol. 40, No. 2, pp. 160, 2008.

[4] J. Huber, J. Steimle, and M. Mühlhäuser, "Toward More Efficient User Interfaces for Mobile Video Browsing: An In-Depth Exploration of the Design Space," in Proceedings of ACM Multimedia (ACM MM), 2010.

[5] T. Karrer, M. Wittenhagen, and J. Borchers, "PocketDRAGON: A Direct Manipulation Video Navigation Interface for Mobile Devices," in Proceedings of the 11th International Conference on Human-Computer Interaction with Mobile Devices and Services (mobileHCI), 2009.

[6] M. S. Lew, N. Sebe, C. Djeraba, and R. Jain, "Contentbased Multimedia Information Retrieval: State of the Art and Challenges," ACM Transactions on Multimedia Computing, Communications and Applications, Vol. 2, No. 1, pp. 1-19, 2006.
[7] J. Martin and C. Trummer, "SCALEX - A Personalized Multimedia Information System for Museums and Exhibitions," in Proceedings of 1st International Conference on Automated Production of Cross Media Content for Multi-Channel Distribution (AXMEDIS), pp.299-302, 2005.

[8] S. Potter, R. Baratto, O. Laadan, L. Kim, and J. Nieh, "MediaPod: A Personalized Multimedia Desktop in Your Pocket," in Proceedings of 11th IEEE International Symposium on Multimedia, 2009.

[9] N. Sebe and Q. Tian, "Personalized Multimedia Retrieval: The New Trend?" in Proceedings of the 9th ACM SIGMM International Workshop on Multimedia Information Retrieval (ACM MIR), 2007.

[10] iCloud. https://www.icloud.com/.

[11] iMovie. http://www.apple.com/tw/ilife/imovie/.

[12] iPhoto. http://www.apple.com/tw/ilife/iphoto/. 